\title{
Psychological Well-Being among Nursing Students
}

\author{
Ali Sabri Radeef Al-Ani \\ Department of Psychiatry, Kulliyyah of Medicine, International Islamic University \\ Malaysia.
}

Introduction: Nursing students are subjected to a variety of stressors during their study. Severe and prolonged stressors may affect the psychological well-being in the form of depression, anxiety and stress which may affect students' academic performance, physical health and quality of life. Therefore, this study aimed to determine the rate and severity of depression, anxiety and stress symptoms and also to assess the relationship between these symptoms with stressors faced by the students. Materials and method: A sample of 174 nursing students from International Islamic University Malaysia participated in this study. Depression Anxiety, Stress Scale (DASS-21) was used to assess the psychological well-being by determining the prevalence and severity of depression, anxiety and stress (DAS) symptoms. The sources of stressors were identified by giving the students a list of the most possible source of stressors which were chosen depending on previous studies, and then the severity of stressors and their relationship with these symptoms were assessed. Results: The overall prevalence of depression, anxiety and stress symptoms was $51.7 \%, 81.6 \%$ and $43.1 \%$ respectively but it was found that $13.2 \%, 44.3 \%$ and $10.3 \%$ of nursing students have clinically significant depression, anxiety and stress respectively. No significant differences between the gender and age of students in relation to DAS symptoms. Regarding the source of stressors, the top five stressors decided by the students were fear of failing, examination and grades, study pressure and obligations, fear of unemployment after graduation and academic overload. Conclusion: Depression, anxiety and stress symptoms are present among nursing students which require early intervention. Academic factors can be considered as sources of stressors that may precipitate emotional disturbances among the nursing students. 